\title{
Toxic Epidermal Necrolysis: A Case Report on its Ophthalmic Complications and Management
}

\author{
Urmil Chawla $^{1}$, Nisha Bura ${ }^{2 *}$, Vandana Sharma ${ }^{3}$, Richa Sharma ${ }^{4}$, Chugh JP ${ }^{5}$ \\ ${ }^{1}$ Professor, Regional Institute of Ophthalmology, Pt. B.D. Sharma PGIMS Rohtak, Haryana, India \\ ${ }^{2}$ Resident, Regional Institute of Ophthalmology, Pt. B.D. Sharma PGIMS Rohtak, Haryana, India \\ ${ }^{3}$ Senior Resident, Regional Institute of Ophthalmology, Pt. B.D. Sharma PGIMS Rohtak, Haryana, India \\ ${ }^{4}$ Resident, Regional Institute of Ophthalmology, Pt. B.D. Sharma PGIMS Rohtak, Haryana, India \\ ${ }^{5}$ Senior Professor, Regional Institute of Ophthalmology, Pt. B.D. Sharma PGIMS Rohtak, Haryana, India
}

DOI: $10.36348 /$ sjmps.2020.v06i08.003

| Received: 02.08.2020 | Accepted: 16.08.2020 | Published: 22.08.2020

*Corresponding author: Nisha Bura

\section{Abstract}

Steven-Johnson syndrome (SJS) and toxic epidermal necrolysis (TEN) are potentially life-threatening mucocutaneous diseases characterized by hypersensitivity to medication and infections. Inspite of complete recovery from skin problems without sequelae, survivors can land with serious ocular complications culminating in blindness irrespective of intensive local and systemic therapy. We are describing the clinical course and treatment of a patient with TEN who developed bilateral ankyloblepharon after recovery from the acute attack. Amniotic membrane was applied to the ocular surface of the patient after correcting the ankyloblepharon. Amniotic membrane transplantation (AMT) has been proven to be an effective mode of treatment for severe ocular surface and eyelid inflammation as it decreases the risk of ocular and visual sequelae. Early detection of complications and aggressive treatment of the same are essential for preservation of vision.

Keywords: Amniotic Membrane Transplantation Stevens-Johnson Syndrome, Toxic Epidermal Necrolysis.

Copyright @ 2020: This is an open-access article distributed under the terms of the Creative Commons Attribution license which permits unrestricted use, distribution, and reproduction in any medium for non-commercial use (NonCommercial, or CC-BY-NC) provided the original author and source are credited.

\section{INTRODUCTION}

Stevens-Johnson Syndrome (SJS) and Toxic Epidermal Necrolysis (TEN) are rare diseases of the skin and mucous membranes characterized by acute blistering similar to that of a partial-thickness skin burn. However, the body surface area involvement in TEN is more severe than SJS [1, 2]. Ocular involvement is common and marked with significant incidence of dry eye, pain, photophobia and eventually loss of sight [3, 4]. Severe cases present with membranous conjunctivitis and sloughing of the epithelium from the eyelids, conjunctiva, and cornea. The raw surfaces can adhere to each other leading to keratinized mucosal surfaces, symblepharon, and scarred lid margins with entropion, trichiasis, distichiasis and ankyloblepharon.

Limbal stem cell failure and corneal scarring or infection may result from the acute inflammation. An abnormal tear film and chronic blink-related microtrauma can further add to the insult [5, 6]. Cryopreserved amniotic membrane exhibits potent antiinflammatory and anti-scarring effects [7]. It has a role in improving the visual outcomes, reducing the symptoms of dry eye and decreasing the severity of scarring in ocular surface and eyelids in patients with SJS or TEN. It also improves the severity of ocular surface inflammation [8].

\section{Case Report}

A 35 years female who had been prescribed trimethoprim-sulfamethoxazole for a lower extremity infection by her general physician. She developed a fever of $103^{\circ} \mathrm{F}$ thereafter, which was accompanied by conjunctival injection. An erythematous rash involving $>60 \%$ body surface area with bullae involving $<10 \%$ of the body surface area was present (Figure 1). Her symptoms worsened inspite of discontinuation of the causative medication. She was admitted to the Dermatology department of tertiary care hospital where she was diagnosed with TEN. Ophthalmological consultation was requested given her ocular involvement. 


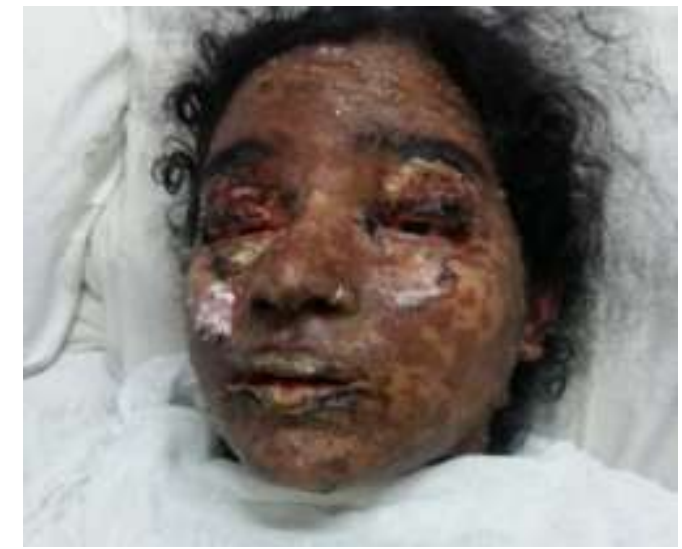

A

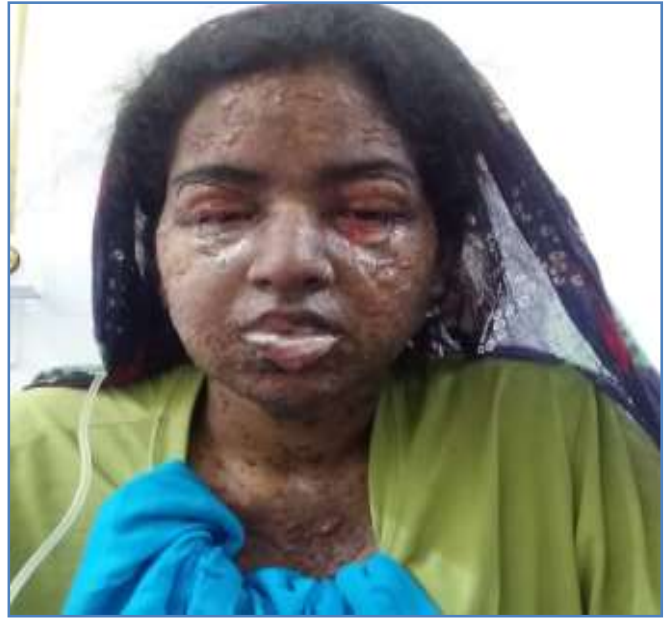

B

Fig-1a, b: Acute phase of TEN.

On ocular examination there was mild, diffuse injection of the bulbar conjunctiva in both eyes. The palpebral conjunctiva was markedly injected. However, fluorescein staining done to evaluate the extent of corneal and conjunctival epithelial defects showed no such lesions. Although there are no standard guidelines for treatment of the same, a combination of topical corticosteroids and antibiotics along with frequent lubricating drops were started. Care was also taken to sweep the fornices regularly to prevent any adhesions. After few months, when her acute phase had resolved she presented to eye OPD with complaints of foreign body sensation, mild difficulty in lid closure and blinking, and also slight restriction of ocular motility.

On ocular examination, a thin sheet of skin was observed at the medial canthus joining the upper and lower lids, in both eyes. Diagnosis of bilateral ankyloblepharon at the medial canthi (Figure 2) was made and after a week of topical antibiotic and lubricating drops, surgical correction was done.

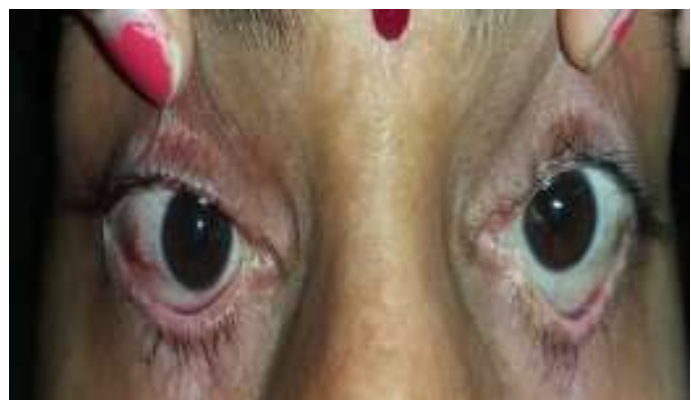

$2 \mathrm{a}$

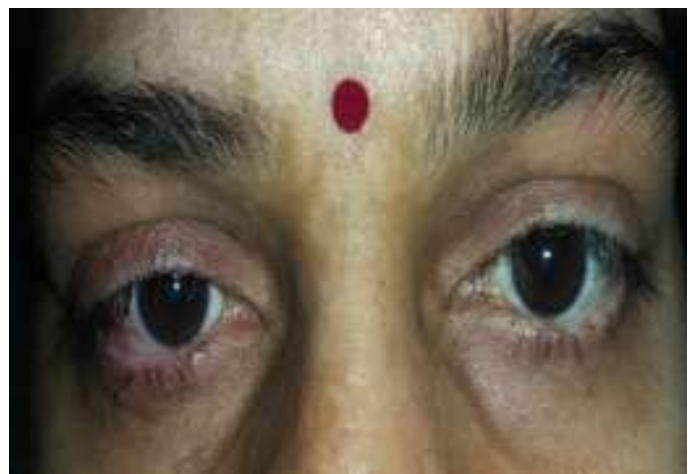

$2 b$

Fig-2a, b:-Presentation of the patient with both eyes medial canthus ankyloblepharon

\section{Surgical Technique}

After excluding other mucosal involvement, patient was taken up for ankyloblepharon release. Under peribulbar anaesthesia, after applying the lid speculum and stay suture, the ankyloblepharon near medial canthus was separated using surgical blade. The irregularities at the lid margin were trimmed and symblepharon adhesions were also released. Amniotic membrane was applied over the lid margins, palpebral conjunctiva, and ocular surface, and anchored in place with bolstered fornix sutures, perilimbal sutures, (8-0 vicryl sutures) and a conformer. (Figure 3a-3d) Right eye was operated first followed by left eye 1 week later. A conformer was placed over the eye to keep amniotic membrane in place.

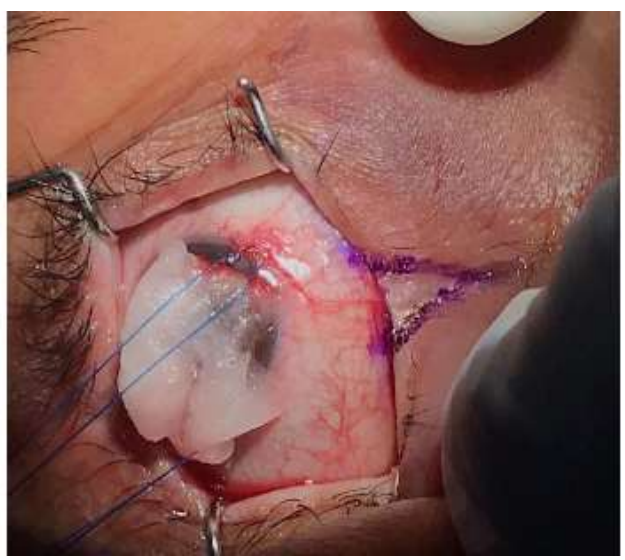

Fig-3a: Exposure and marking of ankyloblepharon 


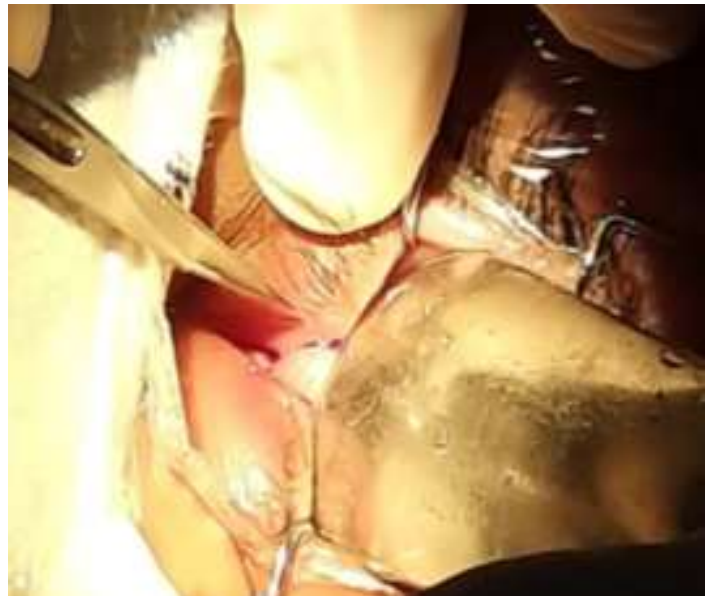

Fig-3b: Excision of the ankyloblepharon tissue after placing spatula beneath for support

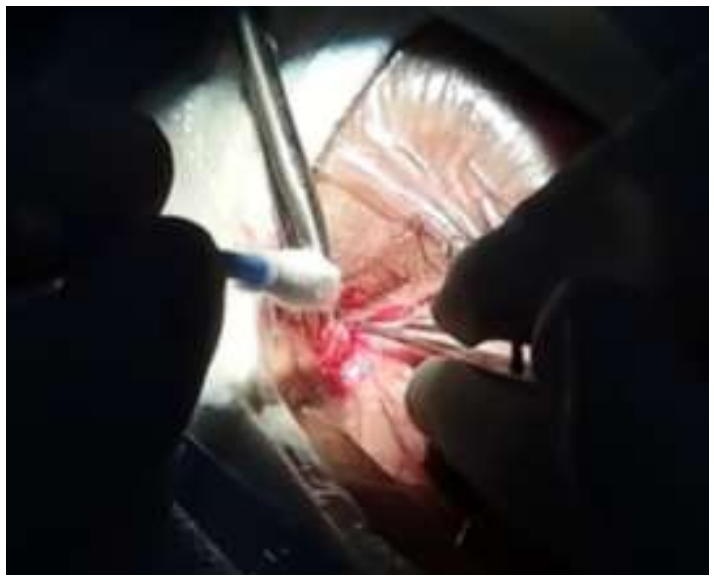

Fig-3c: Release of adhesions

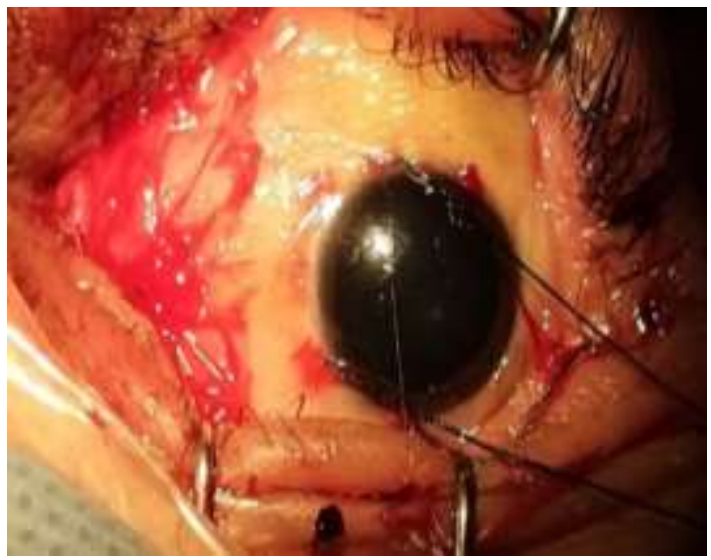

Fig-3d: Amniotic membrane transplantation

Postoperatively the patient received topical antibiotics and anti-inflammatory medications, 4 hourly initially and gradually tapered off over 4-6 weeks, along with liberal use of lubricating eye drops and eye ointment.

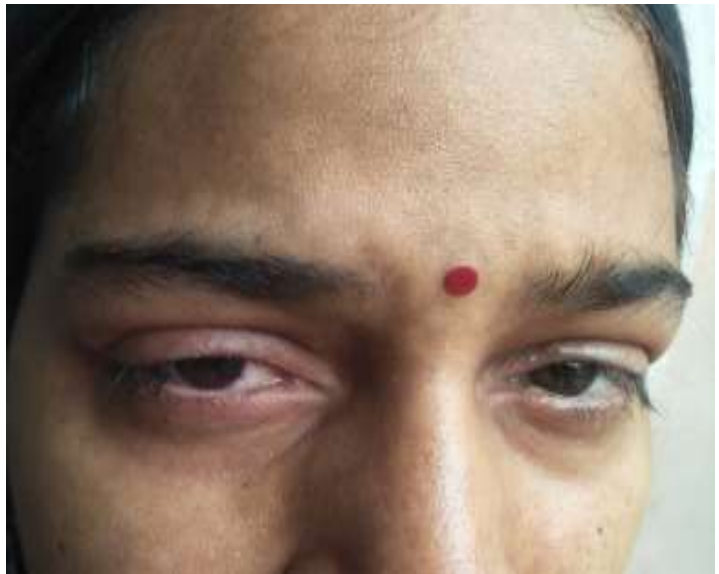

Fig-4: One week postop after B/L ankyloblepharon excision

Postoperative clinical evaluation was performed on day 1 , week 1 , month 1 and month 3 , and every 3 months thereafter (Figure 4, 5). The conformer was removed after 4 wks. At each visit, patient"s comfort was assessed subjectively and ocular examination was done to examine the stability and position of the membrane.

Slit-lamp biomicroscopic evaluation of the ocular surface, including the bulbar, palpebral, and the forniceal conjunctiva and the cornea was done.

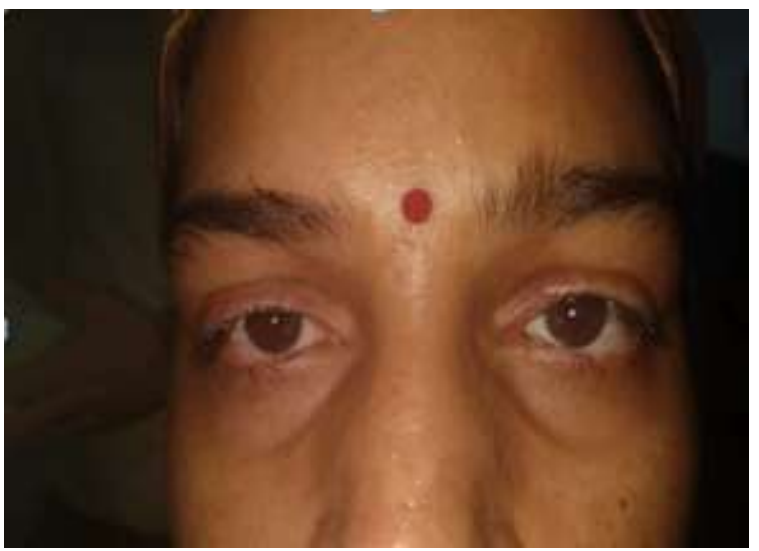

Fig-5: One month Postop after B/L ankyloblepharon excision

\section{DISCUSSION}

Stevens - Johnson syndrome (SJS) and Toxic Epidermal Necrolysis (TEN) are similar conditions characterized by intraepidermal cell death leading to diffuse vesicobullous eruptions. The differentiating feature is the extent of skin detachment. The three diseases can be categorized as:

- $\quad$ SJS : $<10 \%$ total body surface area

- $\quad$ SJS-TEN overlap: 10-30\% total body surface area

- $\quad$ TEN: $>30 \%$ total body surface area 
Up to $90 \%$ of patients present with mucosal involvement. This may involve the ocular surface in as many as $80 \%$ of patients. The incidence of SJS/TEN is estimated to be around 1-7 cases per million per year which is quite low [9].

The exact pathogenesis of SJS/TEN is unknown but seems to occur through cell-mediated keratinocyte apoptosis via the Fas signaling cascade leading to granulysin release. Exposure to certain medications, infections or malignancy can lead to development of this syndrome, though almost one fourth of cases have no known trigger.

Medications such as antibacterial sulfonamides, (trimethoprim/sulfamethoxazole) and anticonvulsants, (e.g. phenytoin) are the leading culprits. Infections are the second most common cause, with especially strong association with Mycoplasma pneumoniae in children. Other infectious causes of SJS/TEN are relatively rare (Table 1 ).

Table-1: Most common causes of SJS/TEN [10]

\begin{tabular}{|c|c|}
\hline $\begin{array}{ll}\text { - } & \text { Allopurinol } \\
\text { - } & \text { Anticonvulsants } \\
\text { - } & \text { Carbamazepine } \\
\text { - } & \text { Lamenytoin } \\
\text { - } & \text { Barbituates } \\
\text { - } & \text { Sulfonamides } \\
\text { - } & \text { NSAIDs }\end{array}$ & $\begin{array}{l}\text { Bacterial } \\
\text { - } \quad \text { Mycoplasma pneumoniae } \\
\text { - } \quad \text { Group A } \beta \text {-hemolytic strep } \\
\text { Viral } \\
\text { - } \quad \text { Cytomegalovirus } \\
\text { - } \quad \text { Herpes simplex virus } \\
\text { - } \quad \text { HIV }\end{array}$ \\
\hline
\end{tabular}

Ophthalmic involvement in SJS often results in a combination of clinical manifestations including conjunctival scarring, symblepharon, ankyloblepharon, dry eye, conjunctivalization of cornea, corneal scar with vascularization, and eyelid malformations, all of which are difficult to treat. The disease can have severe sequelae.

The prognosis is variable and differs with the severity of disease. Long-term ocular complications are serious and affect approximately $60 \%$ of the patients as the ocular involvement is most common and there is risk for severe visual consequences, all patients with SJS/TEN should be urgently evaluated by an ophthalmologist.

\section{Ophthalmologic Management}

There is a lack of studies which have investigated the therapeutic value of topical medications for ocular SJS/TEN. In the absence of any standard treatment guidelines, a combination of topical corticosteroids and antibiotics are prescribed in cases of mild ocular involvement. One retrospective study suggests improved visual outcomes with early topical steroids. In case of more severe ocular involvement, early surgical intervention with amniotic membrane can lead to good outcomes.

Comprehensive management of such cases comprises of the following steps, preferably in sequence [11]:

1. Adequate treatment of dry eye and meibomian gland dysfunction

2. Release of symblepharon/ankyloblepharon, supplementation with AMT aiming at ocular surface reconstruction.

3. Care and correction of eyelid malformations such as entropion, distichiasis, and trichiasis
4. Limbal stem cells transplantation if needed

5. Penetrating keratoplasty

\section{Amniotic Membrane Transplantation (AMT)}

The amniotic membrane is the innermost layer of the placenta. It is mainly comprised of a poorly cellularized stroma with a thick basement membrane.

It has the ability to prevent ocular surface scarring by enhancing epithelialization, reducing inflammation and inhibiting neovascularization. AMT provides a substrate for epithelial cell growth and delivers a complex signaling milieu comprised of immunomodulators and growth factors simultaneously. It can be used as a temporary bandage or a permanent graft [12].

\section{Conclusion}

SJS/TEN may present during acute stage with bilateral conjunctivitis and loss of the ocular surface epithelium. The chronic stage is characterized with cicatricial sequelae and persistent ocular surface inflammation. It leads to a chronically dry surface. The role of AMT in the acute stage is well established and should be performed at the earliest possible opportunity. The use of immunosuppressive and immunomodulatory therapies is controversial. AMT prevents as well as treats symblepharon, ankyloblepharon, eyelid abnormalities, dry eye and corneal disease.

\section{REFERENCES}

1. Roujeau, J. C., Guillaume, J. C., Fabre, J. P., Penso, D., Fléchet, M. L., \& Girre, J. P. (1990). Toxic epidermal necrolysis (Lyell syndrome): incidence and drug etiology in France, 1981- 
1985. Archives of dermatology, 126(1), 37-42.

2. Roujeau, J. C., Kelly, J. P., Naldi, L., Rzany, B., Stern, R. S., Anderson, T., \& Mockenhaupt, M. (1995). Medication use and the risk of StevensJohnson syndrome or toxic epidermal necrolysis. New England Journal of Medicine, 333(24), 1600-1608.

3. Power, W. J., Ghoraishi, M., Merayo-Lloves, J., Neves, R. A., \& Foster, C. S. (1995). Analysis of the acute ophthalmic manifestations of the erythema multiforme/Stevens-Johnson syndrome/toxic epidermal necrolysis disease spectrum. Ophthalmology, 102(11), 1669-1676.

4. Chang, Y. S., Huang, F. C., Tseng, S. H., Hsu, C. K., Ho, C. L., \& Sheu, H. M. (2007). Erythema multiforme, Stevens-Johnson syndrome, and toxic epidermal necrolysis: acute ocular manifestations, causes, and management. Cornea, 26(2), 123-129.

5. De Rojas, M. V., Dart, J. K., \& Saw, V. P. (2007). The natural history of Stevens-Johnson syndrome: patterns of chronic ocular disease and the role of systemic immunosuppressive therapy. British journal of ophthalmology, 91(8), 1048-1053.

6. Di Pascuale, M. A., Espana, E. M., Liu, D. T. S., Kawakita, T., Li, W., Gao, Y. Y., \& Tseng, S. C. (2005). Correlation of corneal complications with eyelid cicatricial pathologies in patients with Stevens-Johnson syndrome and toxic epidermal necrolysis syndrome. Ophthalmology, 112(5), 904-
912

7. Tseng, S. C., Espana, E. M., Kawakita, T., Di Pascuale, M. A., Li, W., He, H., \& Liu, C. Y. (2004). How does amniotic membrane work?. The ocular surface, 2(3), 177-187.

8. Dua, H. S., Gomes, J. A., King, A. J., \& Maharajan, V. S. (2004). The amniotic membrane in ophthalmology. Survey of ophthalmology, 49(1), 51-77.

9. Wang, J.W., Rixen, J.J., Goins, K.M., \& Kitzmann, A.S. (2014). Ocular Manifestations of Stevens Johnson syndrome: 13-year-old female with mucosal and dermatologic eruptions. EyeRounds.org. retrieved August 20, 2014; available from http://EyeRounds.org/cases/192Stevens-Johnson.htm.

10. Hazin, R., Ibrahimi, O. A., Hazin, M. I., \& Kimyai- Asadi, A. (2008). Stevens- Johnson syndrome: Pathogenesis, diagnosis, and management. Annals of medicine, 40(2), 129-138.

11. Honavar, S. G., Bansal, A. K., Sangwan, V. S., \& Rao, G. N. (2000). Amniotic membrane transplantation for ocular surface reconstruction in Stevens-Johnson syndrome. Ophthalmology, 107(5), 975-979.

12. Kang, M. H. (2016). Ocular Manifestations of Stevens-Johnson syndrome and toxic epidermal necrolysis. Hanyang Medical Reviews, 36(3), 174181. 